B-CELL DEVELOPMENT

\section{BLIMP1, BCL6 and B-cell fate}

The differentiation of mature B cells into antibody-secreting plasma cells is controlled by the presence or absence of transcription factors, including B-lymphocyte-induced maturation protein 1 (BLIMP1) and B-cell lymphoma 6 (BCL6), respectively. Recent work has enabled the genes that are targeted by these transcriptional repressors to be identified and has provided an insight into how they interact to control terminal B-cell differentiation.

Forced expression of BLIMP1 drives mature $B$ cells to differentiate into plasma cells. Targets of BLIMP1dependent repression, such as c-MYC, CIITA and PAX5, have been identified previously, but the control of these genes is not sufficient to explain how BLIMP1 regulates terminal B-cell differentiation. To investigate this further, Shaffer and colleagues carried out a DNA-microarray analysis of the changes in gene expression that are induced by BLIMP1, and their findings are now published in Immunity.

Shaffer et al. identified 228 genes that are repressed and 32 genes that are induced in B-cell lines that express BLIMP1 compared with control cells. In many cases, microarray targets were confirmed by independent messengerRNA and protein-expression assays. BLIMP1 was shown to promote B-cell terminal differentiation by turning off the expression of genes that are associated with cell-cycle progression, and DNA synthesis and repair, as well as those that are involved in mature $B$-cell function. These include several transcription factors, such as BCL6 and two newly identified targets, SPI-B and ID3. BLIMP1 also downregulates the expression of genes that are required for immunoglobulin class switching (such as AID, KU70, KU86, DNA-PKCs and STAT6), thereby inhibiting this process. Of the genes that are turned on, of particular interest is the transcriptional activator

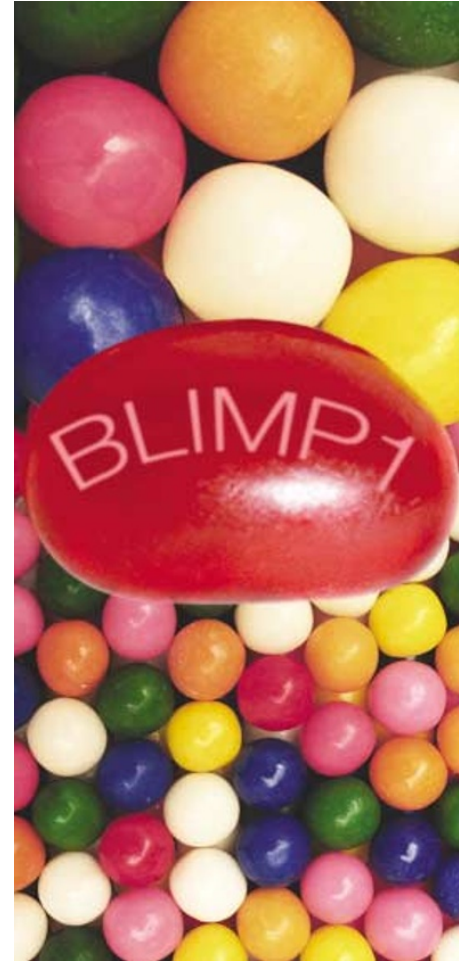

$\mathrm{XBP} 1$, which is a crucial regulator of plasma-cell differentiation. However, the modest increase in XBP1 mRNA in the presence of BLIMP1 indicates that other pathways are also likely to activate XBP1 transcription during plasmacytic differentiation. Therefore, BLIMP1 promotes plasmacytic differentiation by switching off genes that are required for mature B-cell function and switching on plasma-cell genes.

The downregulation of expression of BCL6 by BLIMP1 is consistent with earlier work showing that BCL6 is highly expressed in germinal-centre $\mathrm{B}$ cells, but that it is downregulated as $B$ cells differentiate into plasma cells. BCL6 has been shown previously to repress the transcription of BLIMP1, so Shaffer et al. conclude that a reciprocal regulatory loop might exist whereby BCL6 and BLIMP1 antagonize the expression of each other.

But, how does BCL6 repress the expression of BLIMP1 and other target genes? Work by Vasanwala and colleagues published in The Journal of Immunology has investigated the interactions between BCL6 and the BLIMP1 promoter, and has shown that BCL6 represses BLIMP1 expression by blocking the transcriptional activity of AP1 factors.

Jenny Buckland

(i) References and links ORIGINAL RESEARCH PAPERS Shaffer, A. L. et al. Blimp-1 orchestrates plasma-cell differentiation by extinguishing the mature $\mathrm{B}$-cell gene-expression program. Immunity 17, 51-62 (2002) IVasanwala, F. H. et al. Repression of AP-1 function: a mechanism for the regulation of Blimp-1 expression and B-lymphocyte differentiation by the expression and B lymphocyle differentiation by B-cell lymphoma-6 protooncogene. J. Immunol.
169, $1922-1929$ (2002).

\section{IN BRIEF}

\author{
B-CELL DEVELOPMENT
}

CD22 regulates early B-cell development in BOB.1/OBF.1-deficient mice.

Samardzic, T. et al. Eur. J. Immunol. 32, 2481-2489 (2002)

In the absence of the B-cell-specific transcriptional co-activator BOB.1, mice have reduced numbers of mature B cells due to a block at the transitional B-cell stage. In this study, Samardzic et al. show that expression of the inhibitory co-receptor $\mathrm{CD} 22$ by B-cell precursors is increased in the bone marrow of $B O B \cdot 1^{-/-}$mice. The authors generated $B O B \cdot 1^{-/-} C d 22^{-/-}$double knock-out mice; they show that they have normal B-cell development in the bone marrow and that $\mathrm{Ca}^{2+}$ mobilization in response to $\mathrm{B}$-cell-receptor (BCR) stimulation is restored in mature $\mathrm{B}$ cells. Together, these experiments indicate that the increased expression of CD22 in BOB. $1^{-/-}$mice acts as a brake on BCR signalling, which blocks B-cell development at the transitional stage.

\section{AUTOIMMUNITY}

\section{Cblb is a major susceptibility gene for rat type-1} diabetes mellitus.

Yokoi, N. et al. Nature Genet. 31, 391-394 (2002)

Insulin-dependent diabetes mellitus (IDDM) results from the complex interplay of many genetic and environmental susceptibility factors. Several animals spontaneously develop a disease that is similar to IDDM and they are useful models in which to unravel the complex aetiology of this disease. Previous studies of the Komeda diabetes-prone (KDP) rat have identified two main susceptibiltiy loci - the MHC and $I d d m / k d p 1$ loci. By comparing mouse and rat backcrosses, the authors were able to place $I d d m / k d p 1$ in a $3.0 \mathrm{cM}$ segment, and two genes were then mapped to this locus. One of these genes - Cblb (Casitas B-lineage lymphoma b) - contained a nonsense mutation in KDP rats. The diabetic phenotype of the KDP rat was completely rescued by a wild-type Cblb transgene, which confirms that an absence of Cblb is the prinicipal cause of diabetes in KDP rats.

\section{ASTHMA}

Antigen-specific regulatory $T$ cells develop via the ICOS-ICOS-ligand pathway and inhibit allergy-induced airway hyperreactivity.

Akbari, O. et al. Nature Med. 29 July 2002 (DOl 10.1038/nm745)

Uncontrolled Thelper $2\left(\mathrm{~T}_{\mathrm{H}} 2\right)$ responses in the lung lead to the development of asthma and allergy, so how are they controlled normally? In this study, Akbari and colleagues show that after intranasal exposure to the model antigen ovalbumin, mouse lung dendritic cells produce interleukin-10 (IL-10) and can prime transgenic ovalbumin-specific $\mathrm{T}$ cells in vitro to become IL-10-secreting regulatory $T\left(T_{R}\right)$ cells. When the $T_{R}$ cells were transferred into mice, they markedly inhibited the development of $\mathrm{T}_{\mathrm{H}} 2$ responses and ovalbumin-induced airway hyperreactivity. In addition to IL-10, the development of $\mathrm{T}_{\mathrm{R}}$ cells depended crucially on the ICOS-ICOS-ligand co-stimulatory pathway. 